\title{
Peri-infarct edema leads to overestimation of myocardial salvage in late reperfused myocardial infarction
}

\author{
lacopo Carbone, Marco Francone, Ingo Eitel, Jan Bogaert, Peter Bernhardt, Pier Giorgio Masci, Sven Plein, \\ Matthias G Friedrich ${ }^{*}$ \\ From 16th Annual SCMR Scientific Sessions \\ San Francisco, CA, USA. 31 January - 3 February 2013
}

\section{Background}

In patients with acute myocardial infarction (AMI) the myocardial salvage index (MSI) quantified by Cardiovascular Magnetic Resonance (CMR) is defined as the volumetric difference of myocardial edema, reflecting the perfusion bed, and the infarcted tissue.

While after a coronary occlusion time of more than 8 hours, relevant myocardial salvage is highly unlikely, previous data however showed myocardial edema surrounding the infarcted tissue after prolonged occlusion times. We therefore speculated that peri-infarct edema can be consistently observed in very late reperfused MI, indicating that edema in late reperfused MI does not necessarily reflect myocardial salvage.

\section{Methods}

In 7 tertiary hospitals, 80 patients who underwent PCI between 8 to 24 hours after presentation with AMI were included in the study. The time to reperfusion (TTR) was correlated with infarct size (IS), extent of myocardial edema and MSI. Standard CMR protocols were used to characterize the extent of myocardial edema, infarction, microvscular obstruction (MO) and the apparent myocardial salvage index. A stratified analysis was conducted considering subjects divided in four quartiles of TTR.

\section{Results}

The mean TTR was $706 \pm 238$ minutes. The edematous area was consistently larger than the infarct (mean difference $9.8 \pm 8.3 \%$ ) and the apparent myocardial salvage index was $31.1 \pm 20.1 \%$. There was a strong correlation between the area of infarction and the extent of myocardial edema $(r=0.84, p<0.0001)$. TTR was not correlated with the infarct area $(\mathrm{p}=0.45)$, the extent of myocardial edema $(\mathrm{p}=0.17), \mathrm{MO}(\mathrm{p}=0.65)$ or the myocardial salvage index $(\mathrm{p}=0.19)$.

\section{Conclusions}

In patients with late reperfused myocardial infarction, myocardial edema surrounding the infarcted tissue is a consistent finding. Its extent is not correlated with the time to reperfusion. These data indicate that in late reperfused myocardial infarction: a) Peri-infarct edema is a consistent finding and thus has to be taken into account when assessing myocardial salvage and b) Relevant myocardial salvage appears very unlikely. These findings have significant implications for the quantification of myocardial salvage by CMR and for studies on the "time window" for myocardial revascularization.

\section{Funding}

None.

Published: 30 January 2013

doi:10.1186/1532-429X-15-S1-P209

Cite this article as: Carbone et al:: Peri-infarct edema leads to overestimation of myocardial salvage in late reperfused myocardial infarction. Journal of Cardiovascular Magnetic Resonance 2013 15(Suppl 1): P209. 